\title{
Aprendizagem por meio de jogos e sua aplicabilidade na prática docente
}

\author{
Learning through games and its applicability in teaching practice \\ Aprendizaje a través de juegos y su aplicabilidad en la práctica docente
}

Recebido: 03/10/2021 | Revisado: 12/10/2021 | Aceito: 18/10/2021 | Publicado: 20/10/2021

Roque Ribeiro da Silva Júnior

ORCID: https://orcid.org/0000-0001-9517-6823

Universidade do Estado do Rio Grande do Norte, Brasil

E-mail: roquejunior@alu.uern.br

Lígia Fernanda de Araújo

ORCID: https://orcid.org/0000-0002-7400-1677

Universidade do Estado do Rio Grande do Norte, Brasil

E-mail: fernandafaraujo@alu.uern.br

Marcília Ingrid Lima Barroso Nunes

ORCID: https://orcid.org/0000-0002-5871-3930

Universidade Potiguar, Brasil

Universidade do Estado do Rio Grande do Norte, Brasil

E-mail: prof.marciliabarroso@gmail.com

Antônio Eduardo de Oliveira Silva

ORCID: https://orcid.org/0000-0002-7149-7152

Universidade Potiguar, Brasil

E-mail: eduardohiuga20100@gmail.com

Livia Kallahan Soares Pequeno

ORCID: https://orcid.org/0000-0001-5453-9245

Universidade Potiguar, Brasil

E-mail: liviakallahan@gmail.com

Ana Camila Campelo de Albuquerque Nunes

ORCID: https://orcid.org/0000-0002-2030-8331

Universidade Potiguar, Brasil

Centro Universitário Natalense, Brasil

E-mail: ccampelo32@gmail.com

Paloma Katlheen Moura Melo

ORCID: https://orcid.org/0000-0002-9265-038X

Universidade do Estado do Rio Grande do Norte, Brasil

E-mail: palomakatlheen@hotmail.com

Francisco Medeiros de Azevedo Filho

ORCID: https://orcid.org/0000-0003-2725-1945 Universidade Potiguar, Brasil

Universidade do Estado do Rio Grande do Norte, Brasil

E-mail: medeirosfilhoef@hotmail.com

Francisco Sérgio Lopes Vasconcelos Filho

ORCID: https://orcid.org/0000-0003-3140-0085

Universidade Federal do Cariri, Brasil

E-mail: sergio.vasconcelos@ufca.edu.br

\begin{abstract}
Resumo
Nas últimas décadas, os profissionais da área da saúde estão sendo desafiados pela busca de conhecimento científico baseado em evidências, além de aspectos relacionados com o desenvolvimento de conhecimentos, habilidades e atitudes em relação ao uso da tecnologia da informação na prática profissional se faz necessária na composição das competências desejáveis. Neste contexto, este artigo apresenta um estudo de revisão integrativa da literatura (RIL), que contribui para o processo de sistematização e análise dos resultados de outros estudos independentes sobre o tema abordado. Esta revisão visa sistematizar o conhecimento produzido sobre o impacto da gamificação nos processos de ensino e de aprendizagem. $\mathrm{O}$ uso da gamificação deve seguir o planejamento docente adequado e deve considerar o perfil e as necessidades dos estudantes e adequar-se ao público-alvo, seu objetivo deve ser claro e o domínio tecnológico e das regras do game devem estar claros e concisos. O estudo aponta para a necessidade de apropriação de diversas possibilidades da formulação de novas ações baseadas na aprendizagem por meio da gamificação fomentando novas produções.
\end{abstract}

Palavras-chave: Jogos experimentais; Aprendizagem; Educação.

\section{Abstract}

In recent decades, healthcare professionals have been challenged by the search for evidence-based scientific knowledge, aspects related to the development of knowledge, skills and attitudes regarding the use of information 
technology in professional practice, which is necessary in the composition of competencies desirable. This is an integrative literature review (RIL) study, which contributes to the process of systematizing and analyzing the results of other independent studies on the topic discussed. This review aims to systematize the knowledge produced about the impact of gamification on the teaching/learning process. The use of gamification must follow proper teacher planning and must consider the profile and needs of students and suit the target audience, its objective must be clear and the technological domain and game rules must be clear and concise. The study points to the need for appropriating several possibilities for the formulation of new actions based on learning through gamification, fostering new productions.

Keywords: Games, experimental; Learning; Education.

\section{Resumen}

En las últimas décadas, los profesionales de la salud están siendo desafiados por la búsqueda de conocimiento científico basado en la evidencia, aspectos relacionados con el desarrollo de conocimientos, habilidades y actitudes con respecto al uso de la tecnología de la información en la práctica profesional, que es necesario en la composición de competencias deseables. Se trata de un estudio integrador de revisión de la literatura (RIL), que contribuye al proceso de sistematización y análisis de los resultados de otros estudios independientes sobre el tema abordado. Esta revisión tiene como objetivo sistematizar el conocimiento producido sobre el impacto de la gamificación en el proceso de enseñanza/aprendizaje. El uso de la gamificación debe seguir la planificación docente adecuada y debe considerar el perfil y las necesidades de los estudiantes y adaptarse al público objetivo, su objetivo debe ser claro y el dominio tecnológico y las reglas del juego deben ser claros y concisos. El estudio apunta a la necesidad de apropiarse de varias posibilidades de la formulación de nuevas acciones basadas en el aprendizaje a través de la gamificación, fomentando nuevas producciones.

Palabras claves: Juegos experimentales; Aprendizaje; Educación.

\section{Introdução}

Nas últimas décadas, os profissionais da saúde estão sendo desafiados pela busca de conhecimento científico baseado em evidências. Tal cenário tem despertado interesse para a necessidade do profissional em consumir e produzir conhecimentos que sejam específicos à natureza do seu trabalho (Ercole et al., 2014; Mackavey, Cron, 2019).

Existe uma preocupação atual quanto à qualificação desses profissionais como forma de garantir que seus conhecimentos e habilidades estejam alinhados aos novos paradigmas da atenção à saúde, visto que a morfologia humana é um componente crítico no tocante ao treinamento de graduação em cursos da saúde (Felszeghy et al., 2019; Castro, Gonçalves, 2018; Roche et al., 2018).

Estudos afirmam que aspectos relacionados com o desenvolvimento de conhecimentos, habilidades e atitudes em relação ao uso da tecnologia da informação na prática profissional faz-se necessária na composição das competências desejáveis aos profissionais da saúde (Castro, Gonçalves, 2021; Roche et al., 2018).

Felszeghy et al., (2019) complementa ainda que são necessárias inovações em métodos básicos de ensino de ciências para acompanhar um cenário tecnológico que está em constante mudança.

O uso da tecnologia em sala de aula (como por exemplo os jogos digitais), é apresentada como uma estratégia promissora nos processos de ensino e de aprendizagem, que comprovadamente facilita o envolvimento e a aprendizagem significativa, uma vez que um nível mais alto de motivação intrínseca tem impacto positivo no desempenho do conhecimento (Arruda, Arruda, 2013; Luchi et al., 2017; Ignacio, Chen, 2020; Roche et al., 2018).

É sabido dizer que os jogos e seu sistema de recompensa são poderosas ferramentas potencializadoras do aprendizado em diversas áreas de conhecimento (Azevedo, Bomfoco, 2012; Pescador, 2010).

Em virtude do elevado número de pesquisas sobre a influência dos jogos como ferramenta de ensino, houve um aumento de interesse do uso dos mesmos (Borges et al., 2013; Lee e Doh, 2012; Dominguez et al., 2013).

Estudos apresentados na literatura reforçam resultados que, de acordo com a percepção dos alunos, o uso do jogo educacional melhora a compreensão sobre o conteúdo ministrado (Luchi et al., 2017; Mackavey, Cron, 2019). 
Luchi e colaboradores (2017) afirmam que estratégias de ensino que envolvam jogos têm sido usadas em diferentes cursos da saúde para ensino de diversos conteúdos, como tópicos em obstetrícia, psiquiatria, imunologia, farmacologia e fisiologia.

Essas metodologias estimulam no aluno o desenvolvimento da sua autonomia, raciocínio e pensamento crítico tornando-os protagonistas do seu processo de aprendizagem, favorecendo o desenvolvimento de profissionais críticos, atenciosos e com melhores habilidades de tomada de decisão (Luchi et al., 2017; Felszeghy et al., 2019).

Nas pesquisas de Felszeghy e colaboradores (2019) observamos evidências de que as abordagens que sejam focadas no aluno melhoram o aprendizado e o desempenho acadêmico quando comparamos com as estratégias mais tradicionais com foco no educador, viabilizando com que os educadores busquem implementar técnicas de aprendizagem ativa, incluindo por exemplo a gamificação, objetivando aumentar o interesse dos alunos.

Aprendizagem Baseada em Jogos utiliza o uso dos mesmos para o aprendizado. Uma definição muito utilizada é a gamificação, onde elementos associados ao jogo em atividades não lúdicas proporciona ao aluno envolver-se no processo de aprendizagem. A gamificação efetiva-se quando o professor possibilita algum de tipo de recompensa, meta, feedback, sistema de pontos, assim, conceituando a atividade e possibilitando o envolvimento do aluno sem vinculação a jogos específicos. Os alunos aprendem, não jogando jogos específicos, mas sim, com um jogo que foi idealizado para proporcionar interesse pelo assunto proposto em sala (Osório; Gomes; Valente, 2019; Felszeghy et al., 2019; Roche et al., 2018; Zichermann, 2011).

$\mathrm{O}$ uso da gamificação mostra-se eficaz para envolver os alunos em um ambiente desafiador, agregando valor à experiência de aprendizagem (Mackavey, Cron, 2019), fornecendo uma trilha de dados em tempo real que podendo ainda monitorar matematicamente e descobrir oportunidades para novos insights sobre o aprendizado dos alunos (Roche et al., 2018).

Neste contexto, o objetivo do presente estudo é sistematizar o conhecimento produzido sobre o impacto da gamificação nos processos de ensino e de aprendizagem na área da saúde.

\section{Metodologia}

Este estudo trata-se de uma Revisão Integrativa de Literatura (RIL) que investiga o impacto da gamificação sobre os processos de ensino e de aprendizagem da área da saúde, com abordagem quantitativa. De acordo com Ercole, Melo \& Alcoforado (2014), a finalidade da revisão integrativa é a síntese dos resultados obtidos em pesquisas sobre um tema ou questão, de maneira sistemática, ordenada e abrangente.

A construção deste estudo por cinco etapas sendo estas: elaboração da pergunta norteadora, busca ou amostragem na literatura, coleta de dados, análise crítica dos estudos incluídos, discussão dos resultados. A partir desse método é possível identificar as informações relevantes obtidas nas pesquisas selecionadas com a avaliação, interpretação e síntese do conhecimento (Souza, Silva \& Carvalho, 2010).

As revisões integrativas são elaboradas a partir de questionamentos objetivos e suscintos, a fim de selecionar, esclarecer e analisar em categorias pesquisas pertinentes e relevantes. Optou-se pelo método de Principais Itens para Relatar Revisões sistemáticas e Meta-análises (PRISMA), que consiste em um checklist com 27 itens e em um fluxograma de quatro etapas bem definidas, que auxiliará os pesquisadores a aprimorarem as revisões sistemáticas da literatura com ou sem metaanálises (Galvão et al., 2015).

A primeira etapa da revisão foi a identificação do tema e a questão norteadora da pesquisa a partir da estratégia PICO (acrônimo para P: população/pacientes; I: intervenção; C: comparação/controle; O: desfecho/outcome) Assim, definiu-se: "Qual o impacto da gamificação no processo de ensino/aprendizagem"? 
Na segunda etapa, estabeleceram-se os critérios de inclusão, estes foram: artigos indexados às bases supracitadas estudos primários, disponíveis em idioma: português, inglês ou espanhol, disponíveis na integra e que respondessem à questão norteadora do estudo. Os critérios de exclusão foram estudos do tipo: carta ao editor, resumos submetidos a congressos, revisões narrativas, integrativas e sistemáticas e artigos que não se relacionassem à temática proposta pelo estudo. A busca foi feita nas bases de dados Medline via Pubmed e a Biblioteca Virtual da Saúde (BVS) no mês de junho de 2021 por meio do portal de periódicos da CAPES.

Os Descritores em Ciências da Saúde (DeCS), que traduzem e adaptam o MeSH (Medical Subject Headings), são um vocabulário estruturado, trilíngue (português, espanhol e inglês) e baseados em uma coleção de termos essenciais para acesso a pesquisas relevantes (Pillizzon, 2004). Os Decs/MeSh elencados para a pesquisa são: "Jogos Experimentais" / "Games, Experimental” [Mesh]; “Aprendizagem” / “Education” [Mesh]; "Educação” / "Learning” [Mesh].

Adotou-se a busca de alta sensibilidade na qual a primeira seleção resultou em 835 artigos. Utilizou-se do gerenciador de artigos "Rayyan", com eliminação das duplicatas. A etapa seguinte foi composta pela análise dos artigos por título e resumo, excluindo-se os artigos que não se enquadravam nos critérios de elegibilidade e os que não correspondiam ao tema da pesquisa.

Após a remoção das duplicatas, obteve-se 499 artigos, dos quais apenas 30 foram avaliados para elegibilidade, destes, 21 foram excluídos por não estarem disponíveis na íntegra. Assim, a composição final da revisão foi de n=9 artigos, com a identificação dos artigos a partir dos critérios de inclusão; tipo de revista científica; características metodológicas do estudo e avaliação do rigor metodológico.

Desse modo, as informações extraídas dos artigos foram: título, ano de publicação, autores, objetivo, características metodológicas do estudo, avaliação do risco de viés (Rob 2.0), amostra, variáveis e principais estudos (Quadro 1).

$\mathrm{Na}$ quarta etapa, após a seleção do material a ser analisado, buscou-se explicações aos resultados diferentes e/ou conflitantes, verificou-se a análise dos dados obtidas nos resultados e nas informações evidenciadas por meio dos artigos da amostra da revisão, respondendo às seguintes questões: qual a questão norteadora da pesquisa? A questão da pesquisa foi respondida? e se o estudo atingiu seus objetivos? 
Figura 1: Fluxograma do processo de seleção de estudos.

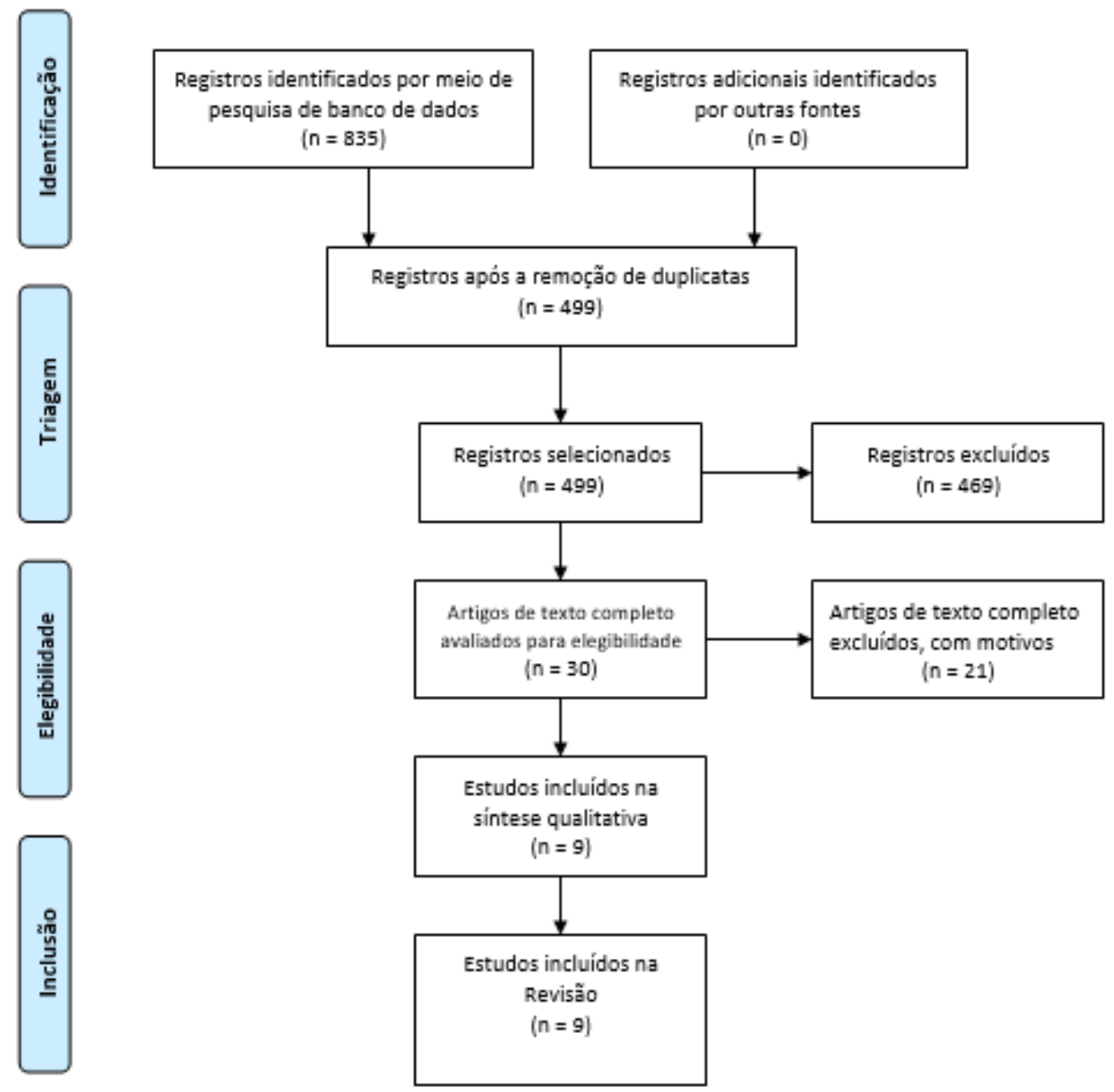

Fonte: Dados da pesquisa.

Na quinta etapa, houve a interpretação dos resultados. Os artigos foram analisados de forma criteriosa, discutindo-se as contribuições desses e compilando os principais resultados em um quadro resumo. Deste modo, após a busca sistemática na literatura, a seleção dos artigos e a organização dos principais achados com a discussão destes foi possível realizar a revisão integrativa.

Na quinta e última etapa, foram apresentadas a revisão/síntese do conhecimento, resultados e discussão dos achados de modo sistematizado, com o intuito de alcançar o objetivo sugerido pelo presente estudo, com a finalidade de propor uma reflexão sobre o uso da gamificação em sua aplicabilidade na prática docente, se há impacto positivo na construção do conhecimento por meio da utilização de tal metodologia.

\section{Resultados}

O resultado da busca gerou uma amostra final de 9 artigos, publicados entre os anos de 2017 e 2021, todas as pesquisas tiveram como local: Faculdades, Centro Universitários e Universidades. Os locais de realização da pesquisa foram: Brasil, Finlândia, Malásia, China, Reino Unido e Estados Unidos da América. Como trata-se de estudos estrangeiros e nacionais, todos estão em língua inglesa, havendo então, uma concentração de lapso temporal entre os últimos 5 anos. Os achados utilizavam a gamificação como cerne norteador, com o intuito de melhorar os níveis de conhecimento, engajamento e 
motivação. Quanto à categoria de estudo utilizado na pesquisa, foram os estudos de corte, quase experimental e mistos. A sistematização dos resultados foi realizada como mostra o Quadro 1.

De tais resultados, surgiram quatro categorias temáticas: motivação e engajamento discente (artigos 1, 3, 5 e 9); “Aumento do Nível de Conhecimento (artigos 1, 2, 3, 4, 6 e 8); “Conhecimento Docente” (artigo 9) e "Construção do Conhecimento Clínico" (artigos 6, 7 e 9). Alguns artigos estão presentes em mais de uma categoria.

Quadro 1: Compêndio de artigos selecionados.

\begin{tabular}{|c|c|}
\hline 1 - Título /Ano & Innovative strategies: Increased engagement and synthesis in online advanced practice nursing education - 2019. \\
\hline Autores & Carole Mackavey; Stan Cron \\
\hline Objetivo & $\begin{array}{l}\text { Avaliar a implementação de estratégias inovadoras e gamificação nas pontuações dos exames de Health } \\
\text { Education Systems Incorporated e aumentar o envolvimento dos alunos e a síntese das informações clínicas. }\end{array}$ \\
\hline Método & Estudo Coorte. \\
\hline $\begin{array}{l}\text { Avaliação Risco de } \\
\text { Viés (RoB 2.0) }\end{array}$ & Risco alto de viés. \\
\hline Amostra & 33 indivíduos. \\
\hline Variáveis & Nível de Conhecimento, Engajamento e Motivação. \\
\hline $\begin{array}{l}\text { Principais } \\
\text { resultados }\end{array}$ & $\begin{array}{l}\text { A gamificação tem um lugar na educação para aumentar o engajamento, a motivação e o aprendizado. Os jogos é } \\
\text { uma ferramenta a ser usada em conjunto com outras mídias disponíveis para um benefício do conhecimento. }\end{array}$ \\
\hline 2 - Título /Ano & $\begin{array}{l}\text { Using online game-based platforms to improve student performance and engagement in histology teaching - } \\
2019 .\end{array}$ \\
\hline Autores & $\begin{array}{l}\text { Szabolcs Felszeghy, Sanna Pasonen-Seppänen, Ali Koskela, Petteri Nieminen, Kai Härkönen, Kaisa Paldanius, } \\
\text { Sami Gabbouj, Kirsi Ketola, Mikko Hiltunen, Mikael Lundin, Tommi Haapaniemi, Erkko Sointu, Eric Bauman, } \\
\text { Gregory Gilbert, David Morton, Anitta Mahonen. }\end{array}$ \\
\hline Objetivo & $\begin{array}{l}\text { O objetivo deste estudo foi o de investigar se os alunos de um curso de histologia médica e odontológica teriam } \\
\text { melhores notas se usassem o software de jogos Kahoot } \AA \text { e os efeitos da gamificação no aprendizado e na } \\
\text { diversão. }\end{array}$ \\
\hline Método & Desenho quase-experimental. \\
\hline $\begin{array}{l}\text { Avaliação Risco de } \\
\text { Viés (RoB 2.0) }\end{array}$ & Risco moderado de viés. \\
\hline Amostra & 215 discentes do curso de odontologia. \\
\hline Variáveis & Nível de Conhecimento. \\
\hline $\begin{array}{l}\text { Principais } \\
\text { resultados }\end{array}$ & $\begin{array}{l}\text { Este estudo dá suporte ao uso de gamificação no ensino de histologia e pode fornecer uma base para a concepção de } \\
\text { um currículo integrado de gamificação em todas as disciplinas da saúde. }\end{array}$ \\
\hline 3 - Título /Ano & Using Kahoot! as a formative assessment tool in medical education: a phenomenological study -2019 . \\
\hline Autores & $\begin{array}{l}\text { Muhd Al-Aarifin Ismail, Anisa Ahmad, Jamilah Al-Muhammady Mohammad, Nik Mohd Rizal Mohd Fakri, } \\
\text { Mohd Zarawi Mat Nor, Mohamad Najib Mat Pa. }\end{array}$ \\
\hline Objetivo & $\begin{array}{l}\text { O objetivo do estudo fenomenológico foi realizado para explorar as vantagens da gamificação por meio do uso } \\
\text { do Kahoot! plataforma de avaliação formativa em educação médica. }\end{array}$ \\
\hline Método & Desenho fenomenológico. \\
\hline Avaliação Risco de & Risco alto de viés. \\
\hline
\end{tabular}




\begin{tabular}{|c|c|}
\hline Viés (RoB 2.0) & \\
\hline Amostra & 36 indivíduos. \\
\hline Variáveis & Nível de conhecimento, engajamento. \\
\hline $\begin{array}{l}\text { Principais } \\
\text { resultados }\end{array}$ & $\begin{array}{l}\text { Os resultados sugerem que Kahoot! as sessões motivam os alunos a estudar, a determinar o assunto que precisa } \\
\text { ser estudado e a ter consciência do que aprenderam. Assim, a plataforma é uma ferramenta promissora para } \\
\text { avaliação formativa na educação médica. }\end{array}$ \\
\hline \multirow[t]{2}{*}{4 - Título /Ano } & Interactive Learning for Nurses Through Gamification - 2021. \\
\hline & Elisabeth Garrison, Sharon Colin, Olivia Lemberger, Maureen Lugod. \\
\hline Objetivo & $\begin{array}{l}\text { O objetivo deste estudo foi o de examinar o efeito da incorporação da gamificação na aquisição de } \\
\text { conhecimento. }\end{array}$ \\
\hline Método & Desenho de estudo quase experimental. \\
\hline $\begin{array}{l}\text { Avaliação Risco de } \\
\text { Viés (RoB 2.0) }\end{array}$ & Risco alto de viés. \\
\hline Amostra & 230 indivíduos. \\
\hline Variáveis & Nível de Conhecimento. \\
\hline $\begin{array}{l}\text { Principais } \\
\text { resultados }\end{array}$ & $\begin{array}{l}\text { Os resultados demonstram a gamificação como uma forma eficaz de aumentar a aquisição de conhecimento } \\
\text { quando comparada aos métodos tradicionais. }\end{array}$ \\
\hline 5 - Título /Ano & Educational Analytics: A New Frontier for Gamification? - 2018. \\
\hline Autores & Cathy Roche, Nancy Wingo, Andrew Westfall, Andres Azuero, Donald Dempsey, James Willig. \\
\hline Objetivo & $\begin{array}{l}\text { Os professores usaram a gamificação como estratégia para aumentar o envolvimento e aprimorar o aprendizado } \\
\text { do aluno. }\end{array}$ \\
\hline Método & Estudo Coorte. \\
\hline $\begin{array}{l}\text { Avaliação Risco de } \\
\text { Viés (RoB 2.0) }\end{array}$ & Risco alto de viés. \\
\hline Amostra & 133 indivíduos. \\
\hline Variáveis & Nível de Conhecimento. \\
\hline $\begin{array}{l}\text { Principais } \\
\text { resultados }\end{array}$ & $\begin{array}{l}\text { Embora seja certamente possível que os pesquisadores não tenham conseguido identificar os efeitos que } \\
\text { realmente existem, estudo mostrou que os alunos de enfermagem não estão apenas abertos a ferramentas } \\
\text { educacionais complementares. }\end{array}$ \\
\hline 6 - Título /Ano & Effect of an educational game on university students' learning about action potentials - 2017. \\
\hline Autores & Kelly Cristina Gaviao Luchi, Luís Henrique Montrezor, Fernanda Marcondes. \\
\hline Objetivo & $\begin{array}{l}\text { O objetivo deste estudo foi o de avaliar o efeito de um jogo educativo que é utilizado para ensinar os mecanismos } \\
\text { dos potenciais de ação em membranas celulares. }\end{array}$ \\
\hline Método & Estudo Coorte. \\
\hline $\begin{array}{l}\text { Avaliação Risco de } \\
\text { Viés (RoB 2.0) }\end{array}$ & Risco alto de viés. \\
\hline Amostra & 36 indivíduos. \\
\hline
\end{tabular}




\begin{tabular}{|c|c|}
\hline Variáveis & Nível de conhecimento e motivação. \\
\hline $\begin{array}{l}\text { Principais } \\
\text { resultados }\end{array}$ & $\begin{array}{l}\text { É importante ressaltar que o estudo avaliou a aprendizagem não apenas considerando a percepção dos alunos, } \\
\text { mas também analisando os escores obtidos na avaliação pós-jogo, bem como o número de erros e questões não } \\
\text { respondidas. Portanto, este estudo representa uma importante contribuição quanto à influência dos jogos } \\
\text { educativos na aprendizagem dos alunos, demonstrando sua eficácia. }\end{array}$ \\
\hline 7 - Título /Ano & $\begin{array}{l}\text { The use of web-based classroom gaming to facilitate cognitive integration in undergraduate nursing students: A } \\
\text { mixed methods study }-2020 \text {. }\end{array}$ \\
\hline Autores & Jeanette Ignacio, Hui-Chen Chen. \\
\hline Objetivo & $\begin{array}{l}\text { Determinar se jogos em sala de aula usando uma plataforma baseada na web podem facilitar a integração } \\
\text { cognitiva para preparar alunos de graduação em enfermagem para a prática clínica. }\end{array}$ \\
\hline Método & $\begin{array}{l}\text { Estudo de métodos mistos de delineamento explicativo sequencial. Um estudo de projeto controlado } \\
\text { randomizado, com conhecimento e desempenho de simulação como medidas de resultado, foi conduzido. }\end{array}$ \\
\hline $\begin{array}{l}\text { Avaliação Risco de } \\
\text { Viés (RoB 2.0) }\end{array}$ & Risco moderado de viés. \\
\hline Amostra & 49 indivíduos. \\
\hline Variáveis & Nível de Conhecimento Cognitivo. \\
\hline $\begin{array}{l}\text { Principais } \\
\text { resultados }\end{array}$ & $\begin{array}{l}\text { O uso de jogos em sala de aula para facilitar a integração de conhecimentos com base em conhecimentos e testes } \\
\text { de desempenho não apresentou resultados positivos. }\end{array}$ \\
\hline 8 - Título /Ano & The use of gamification to teach in the nursing field -2018 . \\
\hline Autores & Talita Candida Castro, Luciana Schleder Gonçalves. \\
\hline Objetivo & $\begin{array}{l}\text { Investigar se a oferta de cursos com elementos de gamificação contribui para a formação de competências em } \\
\text { Informática em Enfermagem; e avaliá-lo com base em critérios de ensino e aprendizagem e estrutura de } \\
\text { conteúdo. }\end{array}$ \\
\hline Método & Pesquisa exploratória, aplicada e de inovação tecnológica, com abordagem qualitativa e quantitativa. \\
\hline $\begin{array}{l}\text { Avaliação Risco de } \\
\text { Viés (RoB 2.0) }\end{array}$ & Risco alto de viés. \\
\hline Amostra & 15 indivíduos. \\
\hline Variáveis & Nível de Conhecimento. \\
\hline $\begin{array}{l}\text { Principais } \\
\text { resultados }\end{array}$ & $\begin{array}{l}\text { Para futuras aplicações de gamificação, sugere-se que, assim como em jogos, o professor explore diferentes } \\
\text { formas de aumentar os níveis de dificuldade à medida que o aluno a vivência. Acredita-se que, para o curso } \\
\text { desenvolvido, as novas experiências devem considerar, por exemplo, o aumento de atividades em cada um dos } \\
\text { Módulos e a condição de que essas atividades sejam gradativamente mais desafiadoras para os alunos. }\end{array}$ \\
\hline 9 - Título /Ano & Gamifying anatomy education - 2018. \\
\hline Autores & Eng Tat Ang, Jia Min Chan, Vik Gopal, Ng Li Shia. \\
\hline Objetivo & $\begin{array}{l}\text { O objetivo da pesquisa foi o de descobrir se a gamificação aumenta a motivação para a aprendizagem } \\
\text { autodirigida (SDL) da anatomia humana entre os alunos do primeiro ano de medicina. }\end{array}$ \\
\hline Método & Estudo Clínico Coorte. \\
\hline $\begin{array}{l}\text { Avaliação Risco de } \\
\text { Viés (RoB 2.0) }\end{array}$ & Risco alto de viés. \\
\hline Amostra & 120 indivíduos. \\
\hline
\end{tabular}




\begin{tabular}{|l|l|}
\hline Variáveis & Nível de Conhecimento. \\
\hline $\begin{array}{l}\text { Principais } \\
\text { resultados }\end{array}$ & $\begin{array}{l}\text { Embora seja positivo, há algumas ressalvas a serem observadas com relação à gamificação, em primeiro lugar, } \\
\text { que depende do tutor. Em conjunto, a gamificação pode representar um novo paradigma para o ensino de } \\
\text { anatomia e um momento oportuno para mudar a cultura predominante no setor de saúde e educação. }\end{array}$ \\
\hline
\end{tabular}

Fonte: Dados da pesquisa.

\section{Discussão}

$\mathrm{O}$ uso da gamificação tem impactado de forma positiva o processo de ensino e aprendizagem. A aplicabilidade prática desse método, vem ganhando espaço e notoriedade quando comparados às metodologias tradicionais de ensino. As constantes transformações tecnológicas e sociais emergem pela necessidade de docentes que dominem não somente o conteúdo programático de uma disciplina, mas que tenham domínio de diferentes abordagens pedagógicas que coloquem o discente no centro dos processos de ensino e aprendizagem (Sá et al., 2017).

Na categoria "motivação e engajamento discente" foi descrito na literatura que o uso da gamificação fomenta o aumento do engajamento e motivação dos alunos para a aprendizagem, frisando a forma ativa de como a ferramenta supramencionada é agudizada em seus benefícios quando implementado a alternativas de ensino convencional e tecnológica.

$\mathrm{O}$ engajamento discente está diretamente relacionado às estratégias utilizadas pelos docentes, nesse contexto a gamificação é um método que ganha importância pela sua eficácia na promoção da interação, quando associado à perspectiva da inclusão do discente como um dos atores que constrói o objeto do conhecimento e o faz sentir-se peça primordial deste processo (Sá et al., 2017).

$\mathrm{Na}$ categoria "aumento do nível de conhecimento" foi descrito na literatura que o efeito da incorporação da gamificação na aquisição de conhecimento melhora os níveis de aquisição de conhecimento dos discente. A ferramenta foi vista como excelente, não somente para a aquisição, mas para a fixação dos conteúdos ministrados que passam a ser apreendidos de maneira significativa.

O trabalho docente busca incessantemente métodos que favoreçam a aprendizagem, o panorama atual afirma que tão somente a apropriação do conhecimento restrito de uma área, não é suficiente para o exercício da prática docente que deve considerar a motivação para o aprendizado, o engajamento, a capacidade de refletir, construir e desconstruir são fundamentais ao ensinar/aprender.

$\mathrm{Na}$ categoria "Conhecimento docente" a literatura traz que o uso das ferramentas tecnológicas tem uma maior efetividade em associação à preparação dos docentes, incluindo a necessidade de capacitação para utilização dos recursos de gamificação, para com isso, conseguir efeitos positivos.

Do ponto de vista da eficácia da gamificação a literatura apresenta autores que atrelam as ascensões positivas da metodologia a outros fatores como experiência do docente com os recursos tecnológicos, o domínio de conteúdo dele. Os estudos de Ignacio e Hui-Chen (2020) evidenciaram a não existência na diferença entre quem fez o uso da ferramenta e aquele que não fez, então com isso a gamificação não apresentou eficiência na melhora do nível do conhecimento, motivação e engajamento dos discentes, semelhante ao ponto de vista de outros autores como Castro \& Gonçalves (2018) e Ang et al., (2018) que apresentam eficiência da gamificação de forma parcial e com direção direta ao profissional que rege os conteúdos, bem como entrelaça com o domínio maior dos conteúdos.

$\mathrm{Na}$ categoria "conhecimento clínico", considerou-se o efeito de jogos educacionais sobre o conhecimento clínico e há apontamentos para a instigação à tomada de decisão profissional, trabalho em equipe, em algumas plataformas específicas e a competitividade entre grupos em busca do conhecimento. Firma-se a ideia de que a práxis promove a união entre a teoria e prática e dão sustentabilidade à construção de competências profissionais desejáveis. 
A gamificação se estabelece como uma metodologia ativa de ensino e aprendizagem que tem como finalidade a busca constante pelo aumento do nível de conhecimento, motivação e do engajamento dos discentes. Há uma diversidade de plataformas digitais utilizadas para realizar a gamificação, com ênfase ao Kahoot@. Os discentes ainda trouxeram à tona a fixação de ideias e conhecimentos por meio da gamificação do ensino, demonstrando que dentro da área da saúde a ferramenta é uma alternativa útil e importante para o ensino aprendizagem.

Há predominância da literatura do uso de gamificação a disciplinas ligadas as profissões da área da saúde.

O presente estudo apresenta limitações no que diz respeito à quantidade de bases de dados elencadas, os resultados da revisão integrativa apontam para estudos em sua maioria com grande risco de viés, onde é apontado a necessidade de ter pesquisas com maior nível de evidência científica.

\section{Considerações Finais}

O objetivo da pesquisa foi atingido, possibilitando a compreensão do impacto da gamificação sobre o processo de ensino e aprendizagem e sua aplicabilidade na prática docente. Os estudos apontam para um impacto positivo sobre o processo de ensino e aprendizagem com aumento do engajamento e da motivação para aprendizagem, do nível de conhecimento e de fixação dos conteúdos quando comparados a métodos de ensino convencional.

O uso da gamificação deve seguir o planejamento docente adequado e deve considerar o perfil e as necessidades dos estudantes e adequar-se ao público-alvo. Os objetivos devem ser claros, e o domínio tecnológico e das regras do game e seus objetivos de contribuição para a aprendizagem do aluno devem estar claros e concisos. O estudo aponta para a necessidade de apropriação de diversas possibilidades da gamificação além do uso do Kahoot®.

A pesquisa contribui para a produção científica, visto que a revisão de literatura pode possibilitar a formulação de novas ações baseadas na aprendizagem através da gamificação fomentando novas produções.

Assim, sugere-se mais investigações acerca do impacto do uso da gamificação no processo de ensino/aprendizagem de docentes com o intuito de obter qualidade e eficiência na formação dos discentes.

\section{Referências}

Arruda, E., \& Arruda, D. (2013). E se a escola virar brinquedo? Perspectivas do lazer e dos jogos digitais na aprendizagem. In MILL, R. R. S. (Org.). Escritos sobre educação: desafios e possibilidades para ensinar e aprender com as tecnologias emergentes, São Paulo, 132-67.

Ang, E. T. Chan, J. M., Gopal, V. \& Shia, N. L. (2018). Gamifying anatomy education. Clinical Anatomy, 31(7), 997-1005. https://pubmed.ncbi.nlm.nih.gov/30168609/.

Bomfoco, M. A. \& Azevedo, V. A. (2012). Os jogos eletrônicos e suas contribuições para a aprendizagem na visão de J. P. Gee. Renote - Novas Tecnologias na Educação - UFRGS, Porto Alegre, 10(3), 2012.

Borges, S. S., Reis, H. M., Durelli, V. H. S., Bittencourt, I. I., Jaques, P. A. \& Isonati, S. (2013). Gamificação Aplicada à Educação: Um Mapeamento Sistemático. In Anais do Simpósio Brasileiro de Informática na Educação, 24(1).

Castro, T. C. \& Gonçalves, L. S. (2018) The use of gamification to teach in the nursing field. Revista Brasileira de Enfermagem, 71(3), 1038-45. https://www.scielo.br/j/reben/a/bGRs38N8BH7GCHYtMFCVrLr/abstract/?lang=en.

Domínguez, A., Sáenz-de-Navarrete, J., de-Marcos, L., Fernández-Sanz, L., Pagés, C. \& Martínez-Herráiz, JJ. (2013). Gamifying learning experiences: Practical implications and outcomes. Computers \& Education, 63, 380-92.

Ercole, F. F., Melo, L. S. \& Alcoforado, C. L. G. C. (2014). Integrative review versus systematic review. Reme: Revista Mineira de Enfermagem, 18(1), 9-10. http://www.reme.org.br/artigo/detalhes/904. DOI: http://dx.doi.org/10.5935/1415-2762.20140001.

Felszeghy, S. Pasonen-Seppanen, S, Koskela, A., Nieminen, P., Harkonen, k., paldanius, k. M. A., Gabbouj, S., Ketola, K., Hiltunen, M., Mikael, L., Haapaniemi, T., Sointu, E., BauMAN, E. B., Gilbert, G. E., Morton, D., \& Mahonen, A. (2019). Using online game-based platforms to improve student performance and engagement in histology teaching. Bmc Medical Education, 19(1), 1-11. https://bmcmededuc.biomedcentral.com/articles/10.1186/s12909019-1701-0.

Galvão, T, F. \& Pansan, T. de S. A. (2015). Principais itens para relatar Revisões sistemáticas e Meta-análises: A recomendação PRISMA*: tradução. Epidemiol Serv Saúde, 24, 335-4. http://scielo.iec.gov.br/scielo.php?script=sci_arttext\&pid=S1679-49742015000200017. 
Research, Society and Development, v. 10, n. 13, e510101321368, 2021

(CC BY 4.0) | ISSN 2525-3409 | DOI: http://dx.doi.org/10.33448/rsd-v10i13.21368

Garrison, E., Colin, S., Lemberger, O. \& Lugod, M. (2021). Interactive Learning for Nurses Through Gamification. Jona: The Journal of Nursing Administration, 51(2), 95-100. https://pubmed.ncbi.nlm.nih.gov/33449599/.

Ignacio, J. \& Chen, Hui-Chen. (2020). The use of web-based classroom gaming to facilitate cognitive integration in undergraduate nursing students: a mixed methods study. Nurse Education In Practice, 46, 102-20. https://www.sciencedirect.com/science/article/abs/pii/S1471595318309612.

Ismail, M. Al-Aarifin et al. (2019). Using Kahoot! as a formative assessment tool in medical education: a phenomenological study. Bmc Medical Education, 19(1), 1-20. https://pubmed.ncbi.nlm.nih.gov/31238926/.

Lee, H., \& Doh, Y. Y. (2012). A Study on the relationship between educational achievement and emotional engagement in a gameful interface for video lecture systems. International Symposium on Ubiquitous Virtual Reality, 34-7.

Luchi, K. C. G., Montrezor, L. H. \& Marcondes, F. (2017). Effect of an educational game on university students' learning about action potentials. Advances In Physiology Education, 41(2), 222-30. https://journals.physiology.org/doi/full/10.1152/advan.00146.2016.

Mackavey, C. \& Cron, S. (2018). Innovative strategies: increased engagement and synthesis in online advanced practice nursing education. Nurse Education Today, 76, 85-8. https://pubmed.ncbi.nlm.nih.gov/30776533/.

Osório, A. J., Gomes, M. J., \& Valente, A. L. (2019). Challenges 2019: Desafios da inteligência artificial, artificial intelligence challenges. Universidade do Minho.

Pescador, C. M. (2010). Ações de Aprendizagem Empregadas pelo Nativo Digital para Interagir em Redes Hipermidiáticas tendo o Inglês como Língua Franca. Dissertação (Mestrado) - Universidade de Caxias do Sul, Programa de Pós-Graduação em Educação.

Roche, C., Wingo, N. P., Westfall, A. O., Azuero, A., Dempsey, D. M. \& Willing, J. H. (2018) Educational Analytics. Cin: Computers, Informatics, Nursing, 36(9), 458-65. https://pubmed.ncbi.nlm.nih.gov/29985195/.

Sá, E. F. D., Quadros, A. L. D., Mortimer, E. F., Silva, P. S. \& Talim, S. L. (2017). As aulas de graduação em uma universidade pública federal:planejamento, estratégia didáticas e engajamento dos estudantes. Revista Brasileira de Educação, 22(70), 625-50. https://www.scielo.br/j/ rbedu/a/JbNdQTJ8bSw3jzpNzKkDQQx/?format=pdf\&lang=pt.

Souza, M. T., Silva, M. D. \& Carvalho, R. (2010) Revisão integrativa: o que é e como fazer. Einstein, 8(1), 102-06. http://dx.doi.org/10.1590/s1679$45082010 \mathrm{rw} 1134$.

Zichermann, G. (2011). Gamification by Design: Implementing Game Mechanics in Web and Mobile Apps. Sebastopol: O'Reilly Media. 